\title{
HYPOTHESES TO EXPLAIN THE OCCURRENCE OF MULTIPLE PEAKS OF DFL IN NERVE CONDUCTION MEASUREMENT
}

\author{
K Siddique-e Rabbani \\ Department of Biomedical Physics \& Technology \\ University of Dhaka, Dhaka-1000, Bangladesh \\ and \\ Bangladesh Institute for Biomedical Engineering \& Appropriate Technology (BIBEAT) \\ email: rabbani@univdhaka.edu
}

\begin{abstract}
Distribution of F-Latency (DFL) is a new nerve conduction parameter obtained using evoked EMG responses, and was introduced by us earlier. We had established that DFL is simply related to the distribution of conduction velocity (DCV) of motor fibres in a peripheral nerve, as an approximate mirror image. Normally DFL has a single peak for healthy nerves, but double, or sometimes, triple peaks of DFL were observed in the median nerve, recording the evoked EMG responses from the Thenar muscle in the palm of the hand. From observation, we also established that such multiple peaks of DFL are associated with Cervical Spondylosis (CS). Double peaks were also observed on the nerves of legs suggesting association with Lumbo-sacral spondylotic neuropathy. The present work puts forward hypotheses to explain the occurrence of double or triple peaks of DFL from median nerve in CS, based on principles of physics, physiology and mathematics, and relate these to observations. This includes observations reported in our earlier work, and also obtained from hundreds of patients tested at a clinic managed by the author. The hypothesis assumes similar DCV with single peaks for all the nerve branches that combine to form the median nerve. Compression or degeneation of one or two of the above nerve branches will result in differential shifts of DFLs, and therefore a combination in the median nerve will give rise to double or triple peaks. Initially the explanation was based on radiculopathy, but the hypotheses evolved when newer neuropathological conditions like myelopathy, and external nerve injury were observed. Finally a generalized hypothesis has been put forward that would cover all these abnormal conditions that affect a peripheral nerve of a limb, both in the hands and the legs. Experiments have been suggested that may test these hypotheses, which, if successful, will lead to a greater understanding of neurophysiology and provide a simple and sensitive diagnostic test through the use of DFL.
\end{abstract}

Keywords: Nerve conduction, Distribution of F-Latency, DFL, Distribution of Conduction Velocity, DCV, Cervical Spondylosis, Lumbo-sacral Spondylosis, Peripheral neuropathy

\section{INTRODUCTION}

\subsection{Basic concepts on DFL and its importance}

A peripheral nerve trunk (or simply nerve) consists of thousands of nerve fibres with varying velocities that can be well described by a Distribution of Conduction Velocity (DCV). If a motor nerve (that cause muscle motion) is electrically stimulated action potentials are generated within the individual fibres which traveling to the connected muscle (orthodromic conduction) elicit a compound muscle action potential, an M-response, that can be recorded using surface electrodes. From the stimulation site the action potentials also travel in the opposite direction (antidromic) to their respective cell bodies located in the spinal cord. In most of the motor nerve cells these antidromic potentials simply die down. However, a few percent of the cell bodies back-fire after a short delay and send fresh action potentials down the nerve fibres to the muscle. These in effect produce a delayed compound muscle action potential called the F-response [1-4] which is very much reduced in amplitude with respect to the M-response because of the small number of fibres involved. This has also been shown to be different from a sensory-motor reflex.

M-responses, obtained with repeated supramaximal stimulation (beyond which the amplitude of response does not increase) at sufficient interval, always have the same shape and amplitude, and occur at the same 
latency (time from stimulation to onset of response). On the other hand, F-responses vary in shape, amplitude and latency on repeated stimulation. The reason is random backfiring in the cell bodies. Which nerve cells will backfire is not previously known, it is entirely a random statistical process. Depending on the conduction velocities of the backfiring nerve cells the combined response that is recorded will have such variations. Sometimes there will be no backfiring, and no F-response at all.

Existing motor nerve conduction methods determine the velocities from the onset of M-responses, or from the shortest F-latency from a set of several F-responses. These obviously give the velocity of the fastest fibres in a nerve trunk, while many diseases and disorders involve the slower fibres to various degrees of which very little is known objectively to date. An ideal diagnosis would involve obtaining the DCV in a nerve trunk giving a clear picture of the pathology. Researchers have been trying to extract DCV in various ways [5,6,7], but none is suitable for a clinical setting. Through work done earlier in our laboratory we have been able to conceive and develop a new method to obtain DCV of motor nerve fibres very easily from multiple F-responses. We simply plotted a statistical frequency distribution of the Flatencies obtained from 20 to 40 F-responses, with intervals greater than one second between stimulations. This interval was necessary to ensure a random character of each response, so that one event does not influence the outcome of the next physiologically. We named the distribution obtained as the Distribution of F-latency (DFL), and provided arguments and experimental verification to show that this can give DCV of motor nerve fibres in a nerve trunk, particularly of those that take part in the Fresponses, simply as its mirror image [8]. Thus a simple clinically suitable method was developed to get an insight into details of neural pathology for the first time.

Diameters of nerve fibres of the same kind are almost directly proportional to their respective conduction velocities [9], therefore diameter distribution from a nerve biopsy may be used to represent the pattern of DCV. From such a nerve biopsy it was shown that the full range of myelinated nerve fibres has a bimodal diameter distribution representing a bi-modal DCV. The two peaks representing A-alpha and Adelta nerve fibres have peak velocities of about $60 \mathrm{~m} / \mathrm{s}$ and $20 \mathrm{~m} / \mathrm{s}$ respectively. Of these, only A-alpha group is present in the motor (efferent) nerves [9, p62-64]. Therefore for normal subjects, only the Aalpha fibres with a single peak in the DCV would be involved in any motor conduction study using evoked potentials. DFL, being a mirror image of DCV as suggested, would therefore have a single peaked pattern for normal healthy nerves as well.

In our initial limited study of DFL from the Median nerves of normal subjects [8] the observed DFLs demonstrated single peaks with the values falling away in both directions, almost like a Gaussian distribution. We have a nerve conduction measurement clinic where DFL is routinely obtained from patients. Over the last 5 years or so, DFL has been performed on hundreds of patients. Many patients have one side affected with some kind of neuropathy while the other side is normal. DFL obtained from the normal sides of these patients, from Median and Ulnar nerves in hands, and from Common Peroneal and Tibial nerves in the leg, demonstrated single peaks. Therefore it seems that normal healthy nerve trunks should demonstrate single peaks in their DFL. This agrees with the above mentioned DCV estimated from diameter distribution. However, the converse may not be true, i.e., DFLs demonstrating single peaks may not always indicate normalcy, as discussed shortly below.

This initial study was carried out on 20 median nerves on both sides of 10 subjects. However double peaks of DFL were observed in 4 cases and triple peaks in one, and all of them reported having diagnosed Cervical Spondylosis (CS), or having symptoms typical of this disorder. Through a retrospective analysis of this study we established this association [10]. This multiple peak of DFL would also mean similar numbered peaks of DCV, and existing knowledge could not give any acceptable physiological explanation to this finding. Therefore, it was thought that looking into the basic causes of CS could provide clues to the observation of double peaks or triple peaks in DFL (or in DCV), and could provide new insight into neural physiology and pathology. Besides, double and triple peaks could provide a good basis for diagnosis of these disorders. 
It was also seen in the initial study that some subjects demonstrated DFL with a distinctive broad peak, sometimes with two small humps, which again mostly happened on one side of a patient who had clear
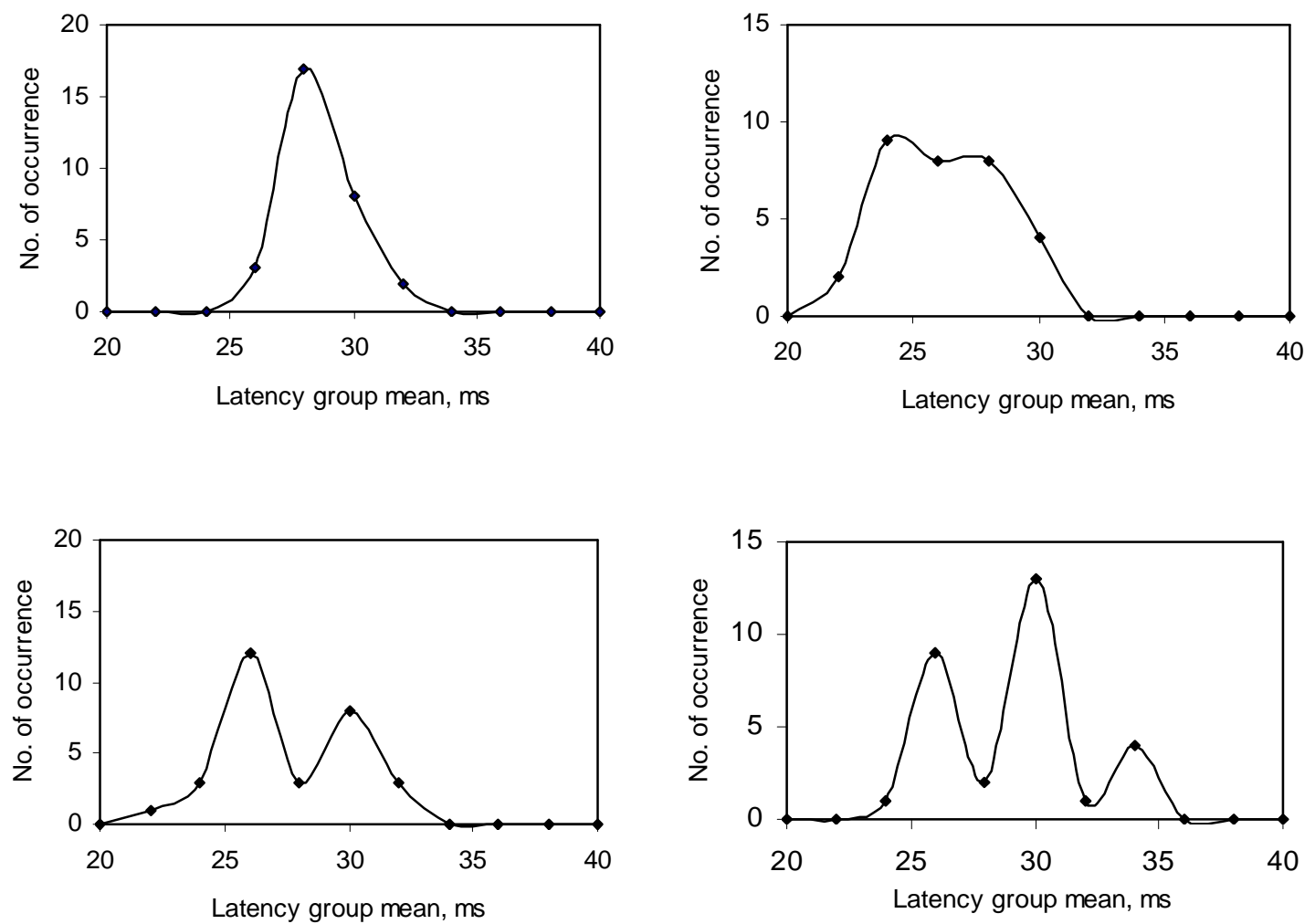

Fig.1: Typical DFL from median nerve of different subjects showing single peak, broad peak, double peak sand triple peaks.

double peaked DFL on the other. In later studies and during routine clinical investigations we found that even some subjects who did not have any complain of CS or did not demonstrate its symptoms had such broad peaks. MRI investigation of one such case showed vertebral disc bulging at C 4-5 and 5-6 levels pressing a little on the spinal cord. In a later follow up after about two years, this subject subsequently demonstrated double peaks of DFL, and started complaining of burning sensations around the shoulder for certain head and shoulder postures. We presume that such broad peaks indicate early stage of CS, for which the patient is yet to feel any effects, i.e., when it is still sub-clinical. Therefore DFL appears to be a very early and sensitive indicator of neuropathy. Typical DFLs with the above features as obtained from human subjects are presented in Fig.1.

During routine clinical work, double peaks of DFL were also observed from Common Peroneal and Tibial nerves in the leg, and in some cases they could be linked to Lumbo-Sacral spondylosis, though this association has not yet been established scientifically. Furthermore, double peaks of DFL were also observed in some cases with stab injuries in the neighbourhood of the nerve concerned. All this information requires a careful in-depth understanding to explain the cause of such observations, which is entirely a new phenomena in nerve conduction investigation. 
Therefore, it appears that explaining the underlying physiological causes for broad peaks and double or triple peaks of DFL of peripheral nerves will be very important from both scientific and clinical points of view, and poses a challenging and interesting research. The present work attempts to put forward several hypotheses to explain such double or triple peaks of DFL. Presenting hypotheses will also allow designing of suitable experiments for their verification, which are also discussed. As mentioned before, we routinely measure DFL in almost all patients who come to a clinic run by us. The DFL patterns that we observed in these patients, although not studied with the necessary scientific vigour, gave a good support to the hypotheses presented here, as well as helped in their evolution.

\subsection{Background information}

Before going into the hypotheses, some background information is summarised below. As mentioned before, the F-wave originates because of random back-firing of a few percent of the cell bodies located in the spinal cord after action potentials reach this end through antidromic conduction along motor nerve fibres in the nerve trunk. The backfiring gives rise to fresh action potentials in the corresponding motor nerve fibres which then travel orthodromically down the nerve trunk to the muscle again. We had argued that since this backfiring involves random recruitment, statistical probability concepts apply. As a nerve trunk contains different number of fibres with different conduction velocities, the probability of getting a particular F-latency will depend on the relative number of nerve fibres having the corresponding conduction velocity. The more fibres with a particular conduction velocity exists, the more chance it will have to contribute to the DFL. Of course we were interested in only the fastest fibre recruited in any stimulation event, by taking the onset F-latency. Here also the statistical probability model would apply. Through this basic argument we related DFL to DCV of motor nerve fibres as its approximate mirror image[8] since latency is inversely proportional to conduction velocity.

In this work we had also shown that for subjects with nerve compression at wrist, a disorder commonly known as Carpal Tunnel Syndrome (CTS), the whole DFL pattern shifts laterally along the time axis, i.e., it is delayed, without significant changes in the pattern. This was convincingly demonstrated for subjects having CTS in one hand and not in the other as shown in Fig.2. Although the conduction velocities of the fibres may remain normal in the whole nerve pathway except at the region of compression, the DFL would shift to longer delays because of the localized compression, or entrapment, and the 'effective DCV' would be shifted to lower velocity values.

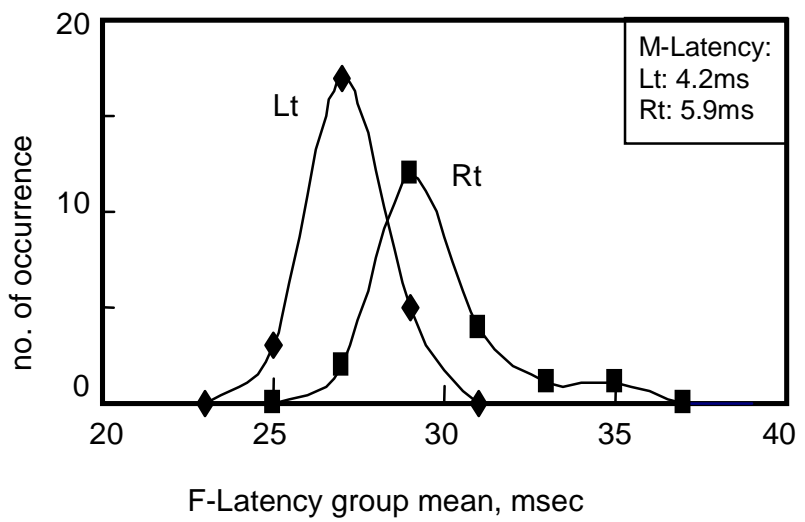

Fig.2: DFL for median nerve for a subject with CTS on right hand, but normal left hand. The M-latency values from the M-responses are quoted in the box (upper right corner). DFL peak shifts by the same amount as that for the M-latencies.

Based on all of the above information coming at

different stages of the work, our hypothesis for the cause of the double or triple peaks in DFL also evolved gradually, and finally we came up with a generalized hypothesis which would be very useful in the design of several experiments for its verification. If the hypotheses are borne out through experiments, these could become important information in the investigation of peripheral neuropathy, both from scientific and clinical points of view. An mentioned before, since CS has been found to be associated with double or triple peaks of DFL, this work may also lead to increased understanding of the underlying physiological causes of CS, and DFL may provide an alternative diagnostic tool for CS. The same may be 
said about Lumbo-Sacral spondylotic neuropathy which is a very common disorder, particularly in the aged population.

\section{HYPOTHESES}

\subsection{Delay shift of DFL in CTS}

Firstly we attempt to explain the delay shift of DFL due to CTS as mentioned above. Carpal tunnel is a channel at the base of the palm through which median nerve, as well as some tendons pass as shown in Fig.3. The carpal ligament covers the tunnel on the top. The nerve almost squeezes though a tight space in this tunnel. Possibly due to the accumulation of fluids in the tunnel, swelling of the tendons, or because of someone having too narrow a channel, the median nerve is compressed, and this causes CTS. In conventional nerve conduction measurement this simply slows down conduction of action potentials while traversing the wrist, and appears as a delay shifted M-response. However, as discussed before, conventional techniques can only relate to the fastest nerve fibres by measuring the latency to the onset of this response, and can say nothing about the degree of slowing in the

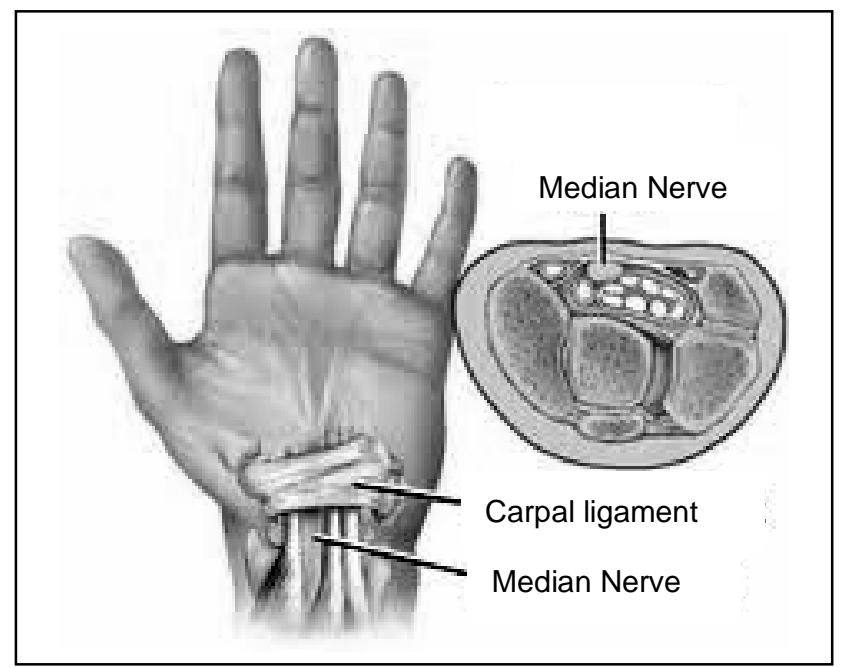

Fig.3: Carpal Tunnel and median nerve in the palm (left), and a cross sectional view (right) showing the tightly placed median nerve. [11] slower fibres.

In our measurement with DFL, the pattern did not change much with CTS. We argue that since all the nerve fibres are immersed in a fluidic environment within a nerve trunk, any compression outside will be transmitted internally through this fluidic environment, and will apply equal pressure on all nerve fibres, because fluid pressure is transmitted to all directions equally, according to Pascal's law in Physics. Therefore, all the nerve fibres will have proportional slowing of conduction velocity due to this compression, and the pattern of DFL will not change significantly. Of course if CTS sustains for a long period, nerve fibres may get degenerated, then the pattern may change. Through the above observation we also hypothesise that compression on the whole of a peripheral nerve trunk at any point in its path will give rise to a delay shift of DFL, but will not affect the general pattern of DFL.

\subsection{Causes of Cervical Spondylosis (CS)}

CS usually has two main causes. One is called Radiculopathy (CS-R) in which nerve branches coming out of the spinal cord are compressed in the narrow channels or gaps created by the vertebral bones. This compression may be caused by bony growth in the vertebra (osteophyte), or herniation of inter-vertebral disc as shown in Fig.4. Either of these compressions leads to CS, and this condition is known as radiculopathy.

The other cause of CS is Myelopathy (CS-M) in which one side of the spinal cord is directly pressed onto, mostly by a bulging intervertebral disc, as shown in Fig.5. This affects nerve fibres located in the pressed region of the spinal cord. 


\subsection{Explaining double and triple peaks of DFL due to radiculopathy}

For the median nerve DFL is usually obtained from the Thenar muscle (Abductor Policis Brevis, APB) at the base of the thumb, by stimulating the nerve at the wrist. It is also known that nerve branches Cervical

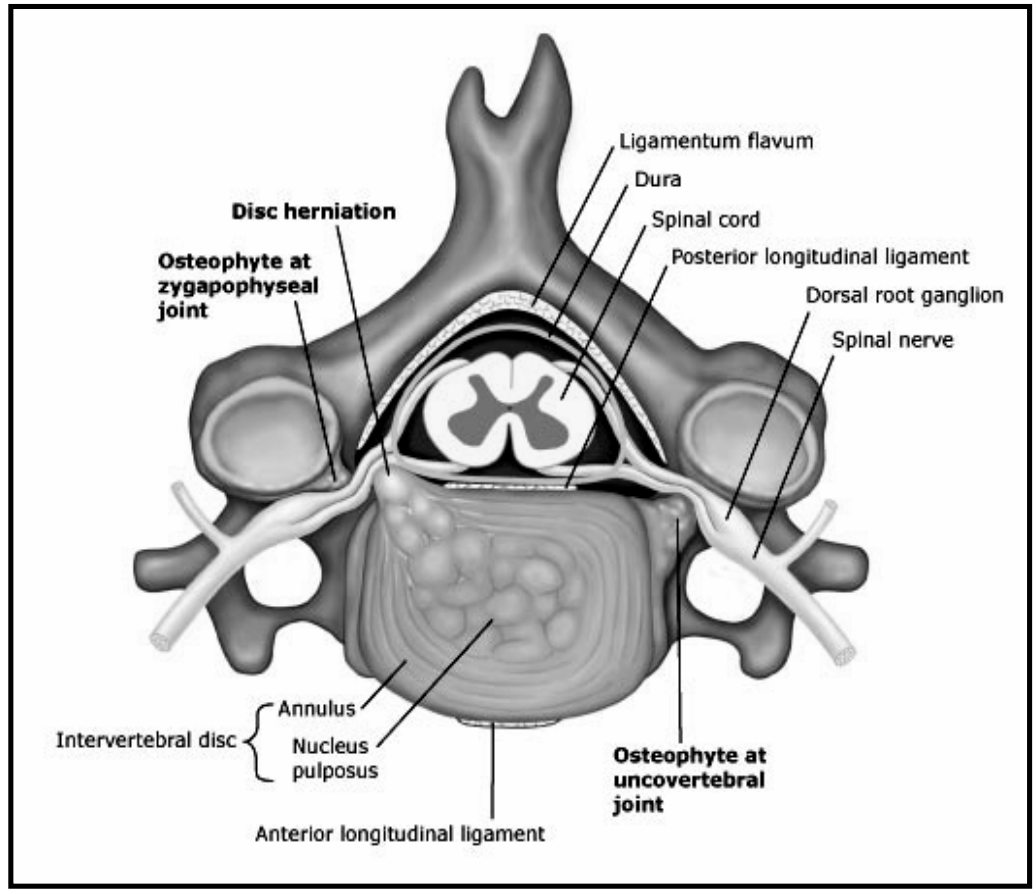

Fig.4: Cross sectional diagram of the vertebra showing nerve branches coming out of the spinal cord at the middle. Also shown are compression on spinal nerve (radiculopathy) due to bony growths (osteophye) at two places, and due to disc herniation [12]

8 (C8) and Thoracic 1 (T1) combine proximal to the Brachial Plexus and contribute to the median nerve to serve the Thenar muscle [13]. Some authors claimed that a nerve branch from $\mathrm{C} 7$ may also contribute [14]. That means three nerve branches, C7, C8 and T1 may contribute to the responses obtained from the APB muscle in the palm of the hand. This information allowed us to present a hypothesis for double or triple peaks of DFL due to radiculopathy as follows.

As mentioned before, for a normal healthy nerve trunk having many fibres, the DCV has a single peak, which gives rise to a DFL with a single peak as well. The same may be expected for the separate DCV of the fibres within each of the nerve branches $\mathrm{C} 7, \mathrm{C} 8$ and $\mathrm{T} 1$ that combine in the median nerve to serve the Thenar muscle. That is, we may expect the relative DCV from each of the nerve branches to be the same, having approximately the same conduction velocity values. This means the DFLs due to the nerve fibres in the three branches will also have similar distributions with approximately the same latency values. The combined DFL due to all the nerve

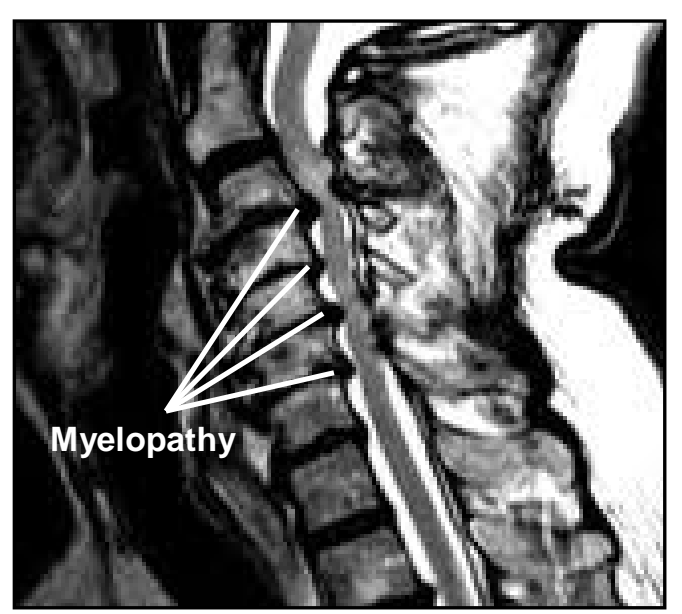

Fig.5: Cervical Spondylotic Myelopathy due to disc bulging, pressing the spinal cord [15] fibres together would be simply a sum of all the three branch distributions, which will also have a similar pattern with a single peak at the same latency value, as shown schematically in Fig.6. This explains why the DFL for a normal healthy nerve has a single peak. 
Now suppose one of the nerve branches, say, $\mathrm{C} 7$ is compressed due to radiculopathy at the vertebral region while the other two, $\mathrm{C} 8$ and $\mathrm{T} 1$ are uncompressed. Therefore, all the nerve fibres within the $\mathrm{C} 7$ branch will be similarly compressed at the point of compression following the same argument as given before for CTS. Therefore, the contribution of nerve fibres from this branch to the measured DFL will have a delay shifted pattern, same as that occurring in CTS. On the other hand, there will be no delay in the DFL for the other two uncompressed nerve branches C8 and T1. Therefore the combined DFL due to

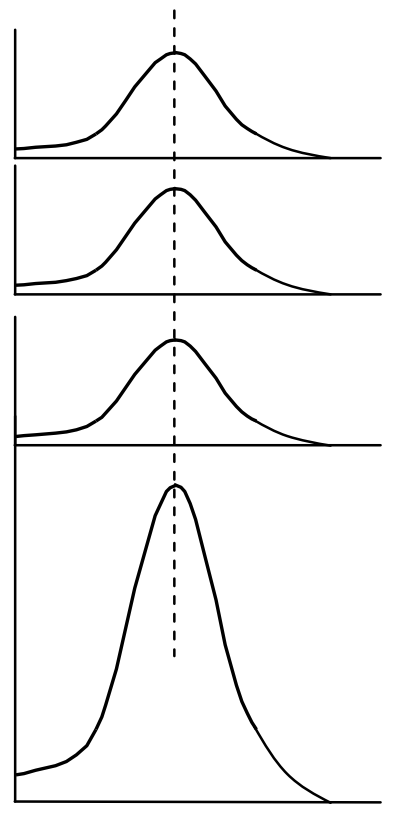

Fig.6: All three nerve branches are normal, i.e., uncompressed. The DFL's for each is the same (top three curves). Combined DFL (bottom curve) has, therefore, a single peak.

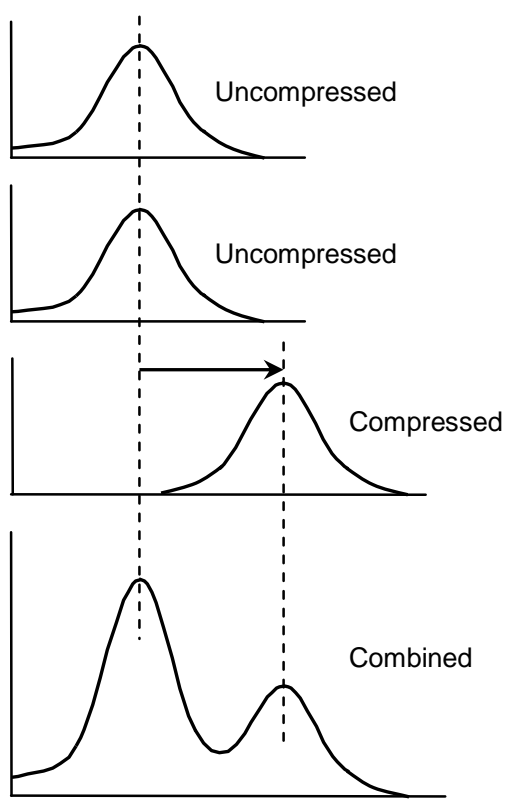

Fig.7: DFL of two nerve branches that are uncompressed (top two curves). Third nerve branch is compressed $\left(3^{\text {rd }}\right.$ from top), giving a latency shifted DFL. Combined DFL (bottom curve) has, therefore, double peak.

nerve fibres from all the three branches will essentially be a sum of the three distributions, and will have a double peak. This is shown schematically in Fig.7. This also will mean that the first uncompressed peak, being from two branches, will be larger than that from the second delayed peak, being from a single branch. This hypothesis was our first to explain the double peak of DFL in CS. It is interesting to note that most of the experimentally observed DFLs with double peaks had the first peak larger than the second one, matching the above explanation. So this hypothesis suggests that compression of any one of the three nerve branches, C7, C8 and T1, will contribute to a DFL with double peaks.

Now if the degree of the above compression is less, as happens in the early stage of radiculopathy, the delay shift of DFL will also be less, and the two peaks of the two distributions may not get resolved in the combined DFL. So they will result in a broad peak as shown in the $2^{\text {nd }}$ distribution in Fig.1. With an increasing degree of compression, the delay will also increase, and two humps may just be noticeable. With further compression, there will be more delay, the two peaks will be resolved and clear double peaks will be noticeable. This probably happened in the two year follow up of a patient mentioned before. Therefore, we hypothesise that a broad peak of DFL indicates an early stage of radiculopathy. As mentioned before, the subject usually does not feel anything at this stage, i.e., it is a sub-clinical phase, and this offers the possibility of early indication of neuropathy. Now, if two of the nerve branches of the 
three $(\mathrm{C} 7, \mathrm{C} 8$ and $\mathrm{T} 1)$ are compressed to different degrees, we may obtain triple peaks, following the same arguments as above. Therefore the same hypothesis also explains the occurrence of triple peaks in DFL as seen in the $4^{\text {th }}$ real life distribution presented in Fig.1.

The same arguments would hold for Common Peroneal nerve and Tibial nerve in the legs due to similar differential compression of the nerve branches in the Lumbo-sacral region of the vertebra.

\subsection{Failure in myelopathy, and presentation of alternative hypothesis}

In order to verify the above hypothesis a subject showing DFL with double peaks of the median nerve was examined using X-ray shadow imaging first. The subject was healthy and young (26 yrs of age) and had no severe complains except some pain in the neck. The X-ray revealed no bony growth at C7, C8 or T1. Therefore, the above hypothesis based on radiculopathy seemed to fail. However, an MRI performed later reported disc bulging and mild pressure directly onto the spinal cord at C4-5 and C5-6 levels, similar to that shown in Fig.5, causing myelopathy. This observation posed a new challenge, to explain the double peak of DFL anew, for which we present the following hypothesis.

Since the spinal cord is a large diameter structure with free space around, this localized pressure will not affect the whole of the cord, as happening in CTS or radiculopathy. We argued that because of the localized pressure on the spinal cord the descending nerve fibres from the brain that lie close to the surface of the spinal cord will become degenerated to some degree. Now some of these descending nerves on the outer side of the spinal cord link to respective cell bodies of peripheral motor nerves that lie at immediately lower vertebral levels through synaptic junctions. Since the body has a nature of shrinking or rejecting organs that are not in use (disuse atrophy), the degeneration of the descending nerves will in turn cause degeneration of the peripheral motor nerves to which they link up. This degeneration will cause a reduction of conduction velocity of these peripheral nerve fibres contributing to a delay shifted DFL. Since the rest of the nerve fibres in the nerve trunk from the other nerve branches are unaffected, the DFL due to these fibres will remain in the original position. Therefore, on combination, double peaks of DFL will result, as in radiculopathy, and shown in Fig.7. Therefore, although myelopathy was observed at C45 or C5-6 level, higher than C7, C8 or T1 nerve branches, the above hypothesis may explain the reason for this myelopathy to affect a part of the median nerve, causing dual peaked DFL.

\subsection{Extension of hypothesis to cover other lesions}

In our nerve conduction clinic, we sometimes observed double peaks of DFL in patients receiving stab injuries in the neighbourhood of a nerve trunk in the arms. Although no rigorous scientific study has yet been taken up on such externally induced neuropathy, we feel that the above hypothesis may be applied to such cases as well. In such cases, we may anticipate degeneration of nerve fibres in a part of the nerve trunk, while the rest remaining intact. Thus the affected or degenerated part of the nerve trunk will contribute to a delay shifted DFL compared to the unaffected part, and when combined, will give rise to a double peak.

Similarly, if a tumour in the spinal canal presses onto the spinal cord, a similar degeneration of peripheral nerve fibres will result contributing to a DFL with double peaks.

\subsection{Generalisation of hypothesis}

All the above point to the fact that if a part of the whole nerve trunk is affected, whatever be the cause, resulting in a reduction of their conduction velocity, double peaks of DFL are likely to be observed. This is schematically represented in Fig.8, Thus DFL can be an effective screening tool for neuropathy. Of course, absence of double peaks does not exclude all neuropathy, such as CTS, but presence of double peak definitely is an indicator of neuropathy. 


\section{SUGGESTION OF EXPERIMENTAL DESIGN}

A test of the above hypotheses has to be done with a few different approaches as follows.

i) Patients with proven cervical or lumbo-sacral spondylotic neuropathy diagnosed using conventional means (clinical symptoms, X-ray, MRI) should be selected. DFL of the affected peripheral nerve should be obtained to see if they demonstrate double peaks. A positive finding will prove the basic hypothesis.

ii) This would be a reverse of the above approach. Here DFL should be obtained from a random selection of subjects, irrespective of neuropathy. Subjects demonstrating broad peak, double or triple peaks of DFL from a particular nerve (say median nerve) should be selected. Spinal X-ray and MRI investigations should be carried out on these subjects to look for abnormalities. If the above hypotheses are valid, these subjects are likely to demonstrate some kind of neuropathy. The same has to be also done for subjects demonstrating double peaks of DFL obtained from ulnar nerve, common peroneal and tibial nerves, and relate the results to X-ray and MRI of the respective appropriate region of the vertebra to look for abnormalities. Positive findings will indicate the sensitivity of DFL in detecting early stages of abnormality.

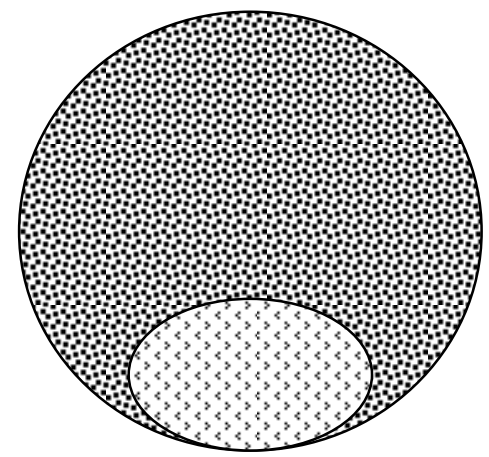

Fig.8: Schematic representation of the general hypothesis: if nerve fibres of a part of a nerve trunk (light dots) have lower conduction velocity than the rest of the fibres in a nerve trunk, then a double peaked DFL will result.

\section{CONCLUSION}

The observation of broad peak, double and triple peaks of DFL in CS was an interesting finding of our earlier work. The effect of CTS in causing a simple delay shift of the whole of DFL without a significant change in its shape was also an important finding. This allowed us to relate it to the effect of compression to the nerve branches near the vertebra through similarity of physical features. In the present work, background knowledge of Physics, Physiology and Statistics have been effectively used in order to explain the experimental observation of broad peak, double and triple peaks of DFL, which are presented as a set of hypotheses. The observation of double peaks initially was through a retrospective analysis of a scientific study on DFL, however, clinical observation of both abnormal and normal limbs of hundreds of patients helped build up confidence for the use of DFL in diagnosis or indication of neuropathy.

To summarise our generalized hypothesis, any entrapment enclosing the whole cross-section of a nerve trunk will lead to a delay shifted DFL, without significant change in its pattern. Any neuropathy causing a reduction of conduction velocity of nerve fibres of one or more segments of the nerve trunk differently, but not the whole, will contribute to broad peak, double or triple peaks.

Therefore, DFL may be used as a first screening tool in the diagnosis of neuropathy. If abnormal delay, broad peak, or multiple peaks are obtained, the patient should be considered for a through test. As mentioned before, people demonstrated broad peaks of DFL even when they did not show any symptoms of neuropathy, and normal clinical check up may not reveal anything. Therefore, DFL will allow identifying cases at sub-clinical stage, at a very early stage of the disorder, making it attractive for patient management.

The hypotheses developed and presented in this paper to explain the broad peak or multiple peaks of DFL will help design experiments for necessary proof. These will also throw more light into the causes of peripheral neuropathy, and will help formulate tests that will help in getting improved diagnosis at an early stage of the disorders. 


\section{ACKNOWLEDGEMENT}

The authors acknowledge the many students, research fellows and technicians working at different times with him in obtaining nerve conduction and DFL data, and to the volunteers and patients who were subjected to these investigations. The author also acknowledge Trauma Centre, Shyamoli, Dhaka for providing space and other logistic facilities for carrying out the clinical work which helped in obtaining a large number of real life observations which helped in developing the hypotheses.

\section{REFERENCES:}

1. Magladery JW, McDougal Jr DB. Electrophysiological studies of nerve and reflex activity in normal man, 1 , Identification of certain reflexes in the electromyogram and the conduction velocity of peripheral nerve fibres. Bull. John Hopkins Hosp. 1950;86:265-290.

2. McLeod JG, Wray SH. An experimental study of the F-wave in the baboon. J. Neurol. Neurosurg. Psychiatry. 1966;29:196-200.

3. Mayer RF, Feldman RG. Observations on the nature of F wave in man. Neurology. 1967;17

4. Fox JE, Hitchcock ER. F wave size as a monitor of motor neuron excitability: Effect of deafferentation. $J$ Neurol Neurosurg Psychiatry. 1987;50:453-459.

5. Hopf HC. Untersuchungen über die Unterschiede in der Leitgeschwindigkeit motorischer Nervenfasern beim Menschen. Dtsch. Z. Nervenheilk. 1962;183:579-588.

6. Ingram DA, Davis GR, Swash M. The collision technique: a new method for measurement of the motor nerve refractory period distribution in man. Electroenceph Clin Neurophysiol. 1987;66:225-234.

7. Harayama H, Shinozawa K, Kondo H, et al. A new method to measure the distribution of motor conduction velocity in man. Electroenceph Clin Neurophysiol. 1991;81:323-331.

8. Rabbani KS, Alam MJ, Salam MA. Frequency Distribution of F-Latencies (DFL) has physiological significance and gives Distribution of Conduction Velocity (DCV) of motor nerve fibres with implications for diagnosis. J of Biol Phys. (Springer), 2007;33:291-303.

9. Kimura J. Electrodiagnosis in diseases of nerve and muscle: Principles and practice, 2nd Ed. Philadelphia, F.A. Davis company; 1989.

10. Alam MJ, Rabbani KS, Possible detection of cervical spondylotic neuropathy using Distribution of Flatency (DFL), a new neurophysiological parameter, BMC Research Notes, 3:112, 2010, <http://www.biomedcentral.com/1756-0500/3/112〉.

11. http://winchellchiropractic.com/chiropractic-conditions/carpal-tunnel/

12. https://www.ssaadvantage.com/images/Vertebra.spinal.png

13. Gray's anatomy. 38th English ed. New York: Churchill Livingstone, 1995:859

14. Harold C. Urschel, Jr and MD Maruf A. Razzuk, Ann Thorac Surg. correspondence 2000;69: 665

15. http://aaspines.com/patients/patients-conditions-cervical.php 